\title{
The Mini Method as an Alternative Approach in Medical Education: A comprehensive Review of the Literature
}

\author{
DhouhaBacha $^{1}$, Lasaad Gharbi ${ }^{2}$, Ghofrane Talbi ${ }^{2}$, Wael Ferjaoui ${ }^{2 *}$, Sahir Omrani ${ }^{1}$ and Bouraoui Saadia $^{1}$ \\ ${ }^{1}$ Department of Pathology, Mongi Slim University Hospital, Faculty of medicine of Tunis, Tunis \\ ${ }^{2}$ Department of General surgery, Mongi Slim University Hospital, Faculty of medicine of Tunis, Tunis
}

*Corresponding author: Wael Ferjaoui, Department of General surgery, Mongi Slim University Hospital, Faculty of medicine of Tunis, Tunis

\section{Introduction}

The "mini method" in medical education is characterized by its duration (25-30 min) and the design of its content [1]. It is divided into minimethods which are intended for teaching (MINIt) and for assessment purposes (MINIa). These mini methods are based on several arguments and data taken from cognitive psychology. Otherwise, they are effective pedagogically [2]. However, they are little used in medical education and their description is dismissed and disparate in the literature. The aim of this study is to carry out a comprehensive review on this mini method in order to codify "a new concept in educational pedagogy». Our work try to define, also, its borders with micro method and classic method (also called macro or long method).

\section{Material and Methods}

A comprehensive literature search was done over a period of 15 years from 1995 to 2015. Databases from Pub med, Google Scholar, The National Library of Medicine (MEDLINE) and the Cochrane Library were searched using the following keywords: mini teaching, mini assessment, medical education, microteaching, learning, pedagogy and the following boolean operators: OR, AND. Articles related to medical education, pharmaceutical education and psycho pedagogy were included in our search. They may be published in English or French language. Editorials are excluded. All articles are read and discussed by all authors of this article. Our study was performed according to the Preferred Reporting Items for Systematic Reviews and Meta-Analyses (PRISMA). References are organized using the ZOTERO software.

\section{Results}

25 articles are selected. The disciplines concerned are medicine, pharmacy, nursing, dentistry and pedagogy. The impact factor of the journals publishing the selected papers varies from 0.2 to 1.5 (SCIMAGO). There are 15 articles describing mini teaching (MINIt)(mini case, mini-chalk talks, mini-reading)) and 10 articles reporting miniassessment (MINIa) (Mini-Clinical Evaluation Exercise (Mini CEX)and mini objective structured clinical examination (MiniOSCE)). In the 1st group there is a difference in the duration of the MINIt which can vary from $10 \mathrm{~min}$ (time usually allocated to micro methods) to $30 \mathrm{~min}$. However, authors are unanimous concerning the content of thisMINIt.The use of the image is reported in these cases. In the second group, the MINIa lasts about $30 \mathrm{~min}$. They are reserved for formative and summative assessment.

\section{Discussion}

The mini method is described initially by Hargie in 1978 (1). It is not a simple extension of micro method format. Indeed, it differs from micro and long methods not only by the duration but also by the content. These differences situate the mini methods exactly between the micro methods and the long methods (Figure 1). The duration of 25-30min of MINIt constitutes the limit of attention of any learner [3]. The participation/ action allow the learner to co-construct his knowledge and the feedback is immediate and personalized. While the main advantage of micro method is the repetition [4] (which is the first principle of all learning), that of mini method is its compilation with other methods increasing, therefore, the motivation of learners. These compilations concern both MINIt (mini chalk talks/bedside) (Figure 2) and MINIa (mini CEX-Direct observation of procedural skills: DOPS) (Figure 3). The principles of the MINIt can be reversed making, so, miniflips like what has been described in the micoflips [5]. There is a lack of grade A evidence proving the educational effectiveness of these mini methods. From an educational point of view, mini methods 
must follow well-established educational goals in perfect alignment between Object-methods-evaluation. On the other hand, The MINIa is reserved for formative and summative assessment and must be followed immediately by a feedback. Therefore, MINIt and MINIa can train into a cycle as described in microteaching [4]. In summary, do not consider the mini, micro and long methods as opposite but rather complementary (which can be adopted alternately). Indeed, the same learning medium (example: clinical case) can have 3 formats: micro (microcase), mini (minicase) and long methods (long case).

\begin{tabular}{|c|c|c|c|}
\hline & micro & $\operatorname{mini}$ & long \\
\hline Duration (min) & $10 \mathrm{~min}$ & $25-30 \mathrm{~min}$ & $45-60 \mathrm{~min}$ \\
\hline number of learners & 5 & 25 & 40 \\
\hline Content ( 3 main sequences) & $\begin{array}{l}\text { 1) Teach } \\
\text { 2) Feedback } \\
\text { 3) Reteach }\end{array}$ & $\begin{array}{l}\text { 1) Teach } \\
\text { 2) Feedback } \\
\text { 3) Action/participation }\end{array}$ & $\begin{array}{l}\text { 1) Teach } \\
\text { 2) Pause } \\
\text { 3) Teach }\end{array}$ \\
\hline Feed back & immediate & immediate & Delayed or absent \\
\hline Transmission of educational message & 1 & 1 to 2 & 3 to 4 \\
\hline
\end{tabular}

Figure 1: Summary table showing the differences between the 3 methods of education.

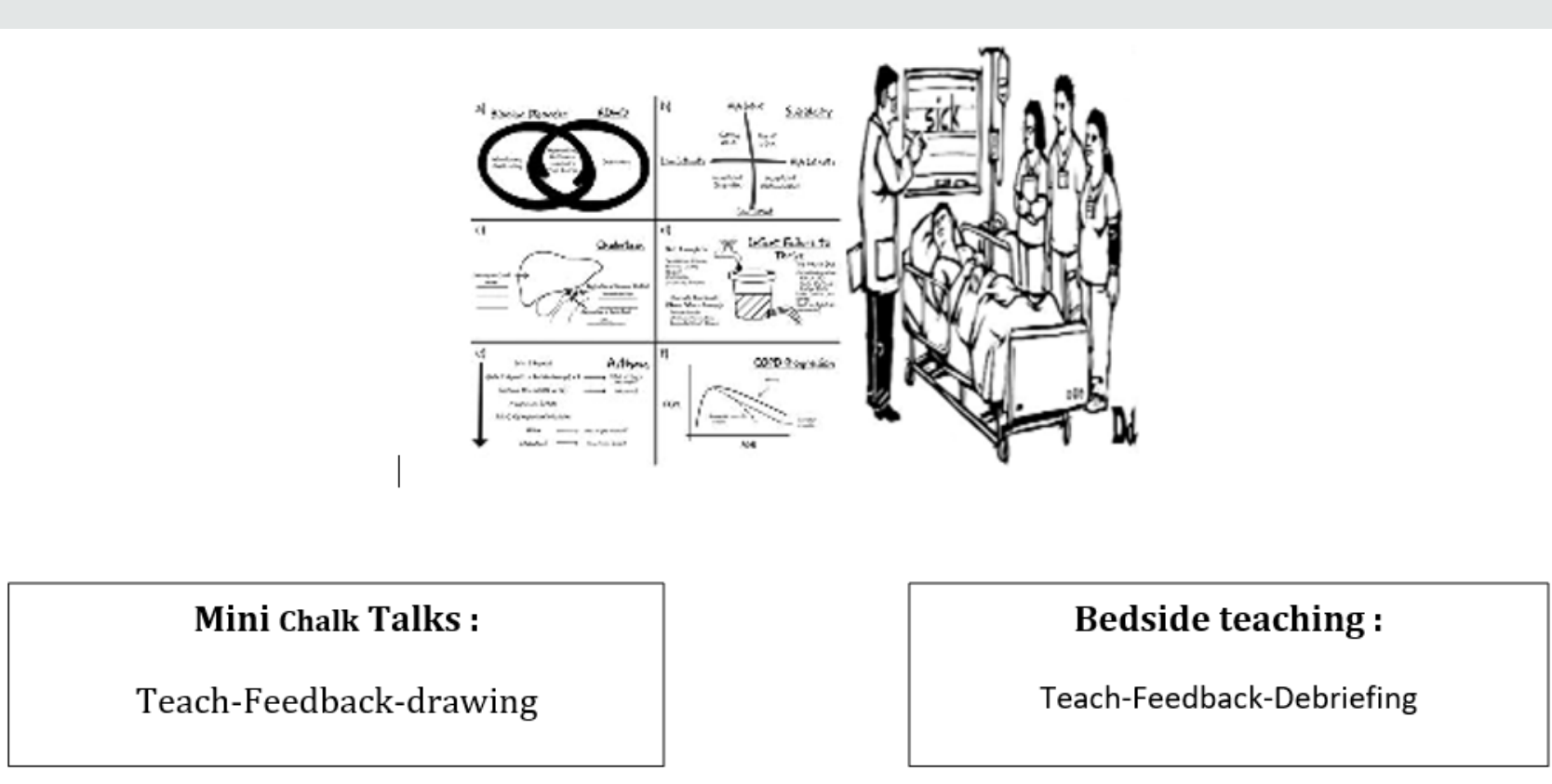

Figure 2: Illustration of miniteaching. 


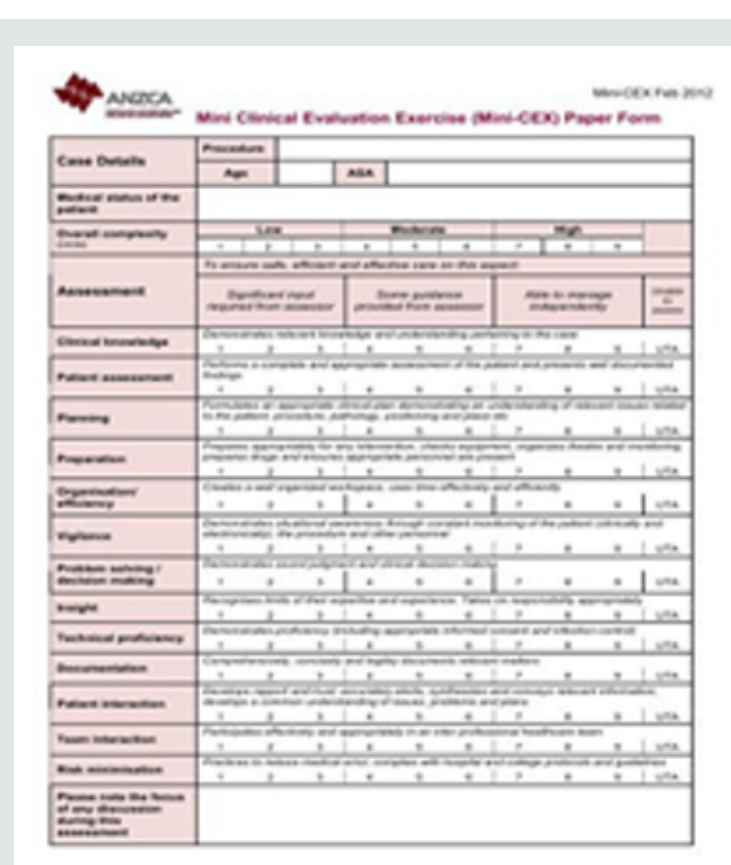

Mini CEX

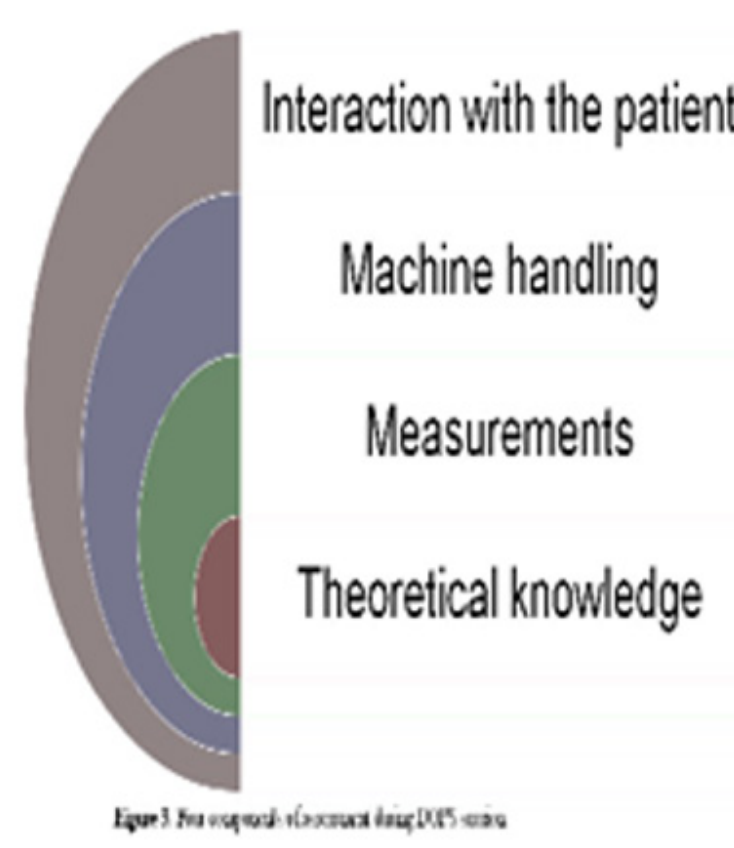

Steps of DOPS
1) Student-patient interaction
2) Interactive correction
3) Feedback

Figure 3: Illustration of mini assessment.

\section{Conclusion}

Minimethode can be adopted as new pedagogical approaches in order to overcome the inadequacy of other conventional methods.

\section{References}

1. Hargie ODW, Dickson DA, Tittmar HG (1978) MINI-teaching: An extension of the microteaching format. British Journal of Teacher Education 4(2): 113-118.
2. Pitt M, Orlander J (2017) Bringing mini-chalk talks to the bedside to enhance clinical teaching. Medical Education Online 22(1): 1264120.

3. Bradbury NA (2016) Attention span during lectures: 8 seconds, 10 minutes, or more? Advances in Physiology Education 40(4): 509-513.

4. Bajaj P, Patil M, Almale B (2014) Microteaching in Medical Education. MVP Journal of Medical Sciences 1: 84.

5. Blanco Á, Martinez-Nuñez M, Borras-Gene O, Sanchez-Medina J (2016) Micro flip teaching-An innovative model to promote the active involvement of students. Computers in Human Behavior 72: 713-723.

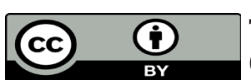

This work is licensed under Creative Commons Attribution 4.0 License

To Submit Your Article Click Here: Submit Article

DOI: $10.32474 /$ OAJOM.2020.03.000168

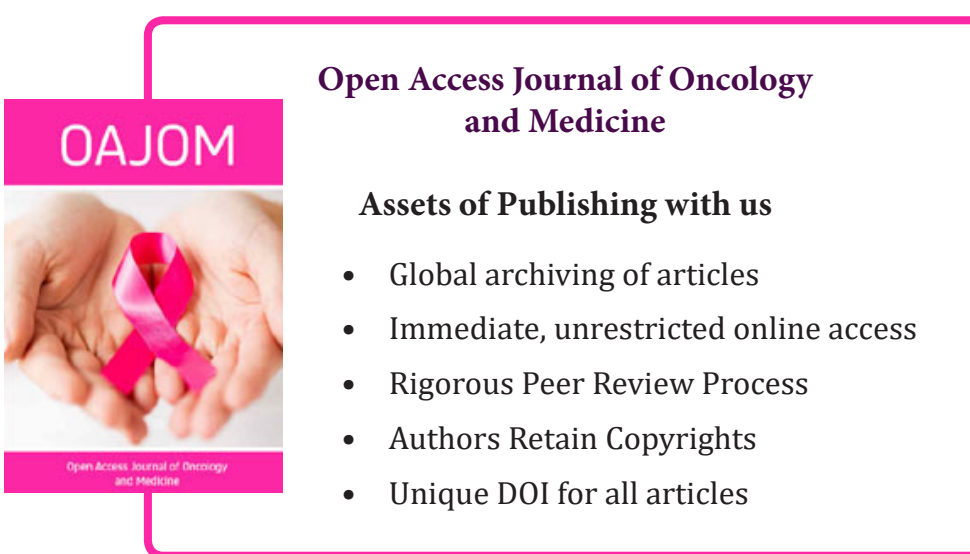

